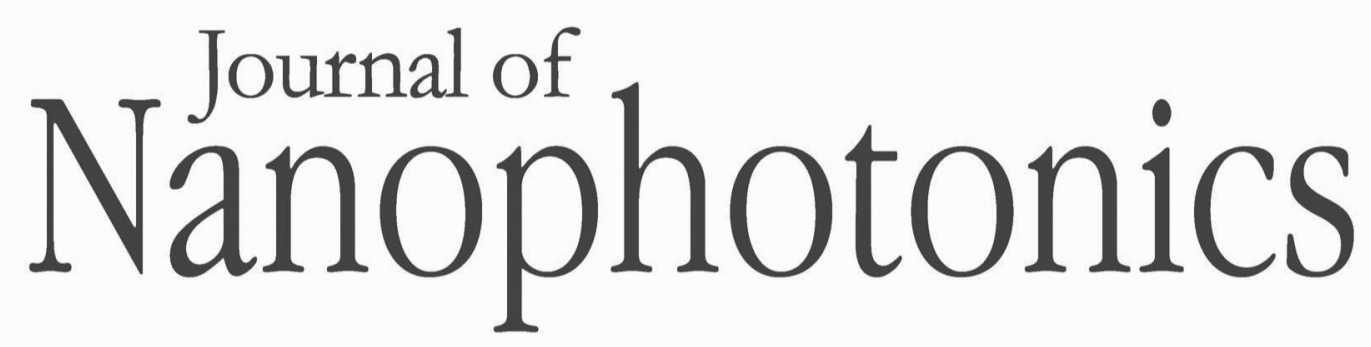

SPIEDigitalLibrary.org/jnp

\title{
Special Section Guest Editorial: Nanostructured Thin Films VI: Latest Developments in Theory and Practice
}

Tom G. Mackay

Yi-Jun Jen

Motofumi Suzuki 


\title{
Special Section Guest Editorial: Nanostructured Thin Films VI: Latest Developments in Theory and Practice
}

\author{
Tom G. Mackay, ${ }^{\mathrm{a}, *}$ Yi-Jun Jen, ${ }^{\mathrm{b}, *}$ and Motofumi Suzukic, $*$ \\ ${ }^{a}$ School of Mathematics and Maxwell Institute for Mathematical Science, University of \\ Edinburgh, James Clerk Maxwell Building, King's Buildings, Edinburgh, EH9 3JZ, \\ United Kingdom; and Department of Engineering Science and Mechanics, \\ The Pennsylvania State University, University Park, Pennsylvania, 16802 \\ ${ }^{b}$ Department of Electro-Optical Engineering, National Taipei University of Technology, \\ No. 1, Sec. 3, Chung-Hsiao E. Road Taipei, Taiwan (106) \\ ${ }^{c}$ Department of Micro Engineering, Kyoto University, Kyoto daigaku-Katsura, \\ Nishikyo-ku, Kyoto 615-8540, Japan
}

In the ever onward march of nanotechnology, thin films with engineered structures at nanometer length scales continue to play central roles. Advances in theoretical and numerical methods together with the establishment of ingenious fabrication and characterization techniques have led to the development of nanostructured thin films with controlled compositions and dimensions, highly specific properties, and several functionalities. This development of increasingly sophisticated nanostructured thin films continues unabated. Clear evidence of the vitality of this field is on display each year at the Nanostructured Thin Films conference, which is a constituent of the SPIE Optics + Photonics annual meeting.

The sixth conference in the Nanostructured Thin Films series took place in San Diego (California, USA) between the 28th and 29th of August 2013. Approximately 44 works were submitted in total. The range of topics that were presented included fabrication, modeling and homogenization, biomimetic thin films, sensing, functional nanostructured thin films, solar cells, characterization, and plasmonics. In addition, an opening keynote session was devoted to the rapidly developing topic of biomimetics. The presented works were spread over nine sessions of oral presentations and a poster session. The presenters came from all parts of the world; they had a variety of backgrounds in engineering and scientific disciplines; and while some were new to our area, others were familiar faces from previous Nanostructured Thin Films conferences.

This special section comprises a set of selected papers that have been expanded from those presented at the Nanostructured Thin Films VI conference or are otherwise closely related to papers presented at this conference. These papers provide a representative snapshot of current research activities in the field of nanostructured thin films and serve to illustrate the diversity of research activities that are ongoing.

\section{Acknowledgments}

The guest editors thank their program committee, all authors for their contributions to this special section, and all those who participated in the Nanostructured Thin Films VI conference and contributed to its proceedings.

Tom G. Mackay is a reader in the School of Mathematics at the University of Edinburgh, and also an adjunct professor in the Department of Engineering Science and Mechanics at the Pennsylvania State University. His current research interests include homogenization, complex materials, and sculptured thin films. He was elected a fellow of SPIE in 2010.

Yi-Jun Jen is a professor at the Department of Electro-Optical Engineering, National Taipei University of Technology. His current research interests primarily involve fabrication of

*Address all correspondence to: Tom G. Mackay, E-mail: t.mackay@ed.ac.uk; Yi-Jun Jen, E-mail: jyjun@ntut.edu.tw; Motofumi Suzuki, E-mail: m-snki@me.kyoto-u.ac.jp 
nanosculptured thin films, novel optical devices based on anisotropic thin films, optical property analysis for subwavelength scale composite material. He was elected as a senior member of SPIE in 2009 .

Motofumi Suzuki is a professor at Kyoto University. He received his bachelor's, master's and doctor of engineering degrees from Kyoto University in 1986, 1988, and 1998, respectively. His current research interests include the optical properties of nanostructured thin films and the synthesis of novel nanostructures. He is a member of SPIE. 